\title{
AVALIAÇÃO DA DISSIPAÇÃO NO AMBIENTE DO RATICIDA BRODIFACUM FORMULADO COMO BLOCO PARAFINADO
}

\author{
SOLANGE PAPINI* \\ RITA DE CÁSSIA B. PRISCO** \\ LUIZ CARLOS LUCHINI $I^{\star \star *}$ \\ VERA LÚCIAT. SAVOY****

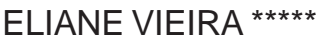 \\ LIA EMI NAKAGAWA******
}

\begin{abstract}
Para o controle químico de roedores na cidade de São Paulo, principalmente ratazanas, são colocados blocos parafinados contendo o ingrediente ativo (i.a.) brodifacum em bueiros sujeitos à ação das intempéries que podem acarretar alterações no i.a. e sua transferência para outros ambientes. Visando verificar essa possibilidade foram montados sistemas experimentais com blocos parafinados, simulando as condições de campo. Também foram colocados 100 blocos em bueiros monitorados semanalmente. Três blocos dos sistemas e dos bueiros foram coletados semanalmente, durante 10 semanas, e analisados por cromatografia a líquido de alta eficiência. Os dados obtidos foram utilizados para a avaliação da dissipação do i.a. Os resultados evidenciaram a estabilidade da molécula sob condições ambientais reais de aplicação, pois $100 \%$ do i.a. foram recuperados após a exposição do bloco. Já, nos sistemas experimentais, cerca de $50 \%$ dos blocos se soltaram do arame de fixação até a quinta semana de exposição e a recuperação do i.a. diminuiu gradativamente ao longo das 10 semanas de estudo, chegando a 50\% da concentração inicial.
\end{abstract}

PALAVRAS-CHAVE: RODENTICIDA - PERSISTÊNCIA; HIDROXICUMARÍNICO; CONTAMINAÇÃO AMBIENTAL.

* Bióloga, Doutora em Ecologia, Secretaria do Verde e Meio Ambiente, Departamento de Controle da Qualidade Ambiental, São Paulo, SP (e-mail: spapini@prefeitura.sp.gov.br).

* Engenheira Agrônoma, Pesquisador Científico, Instituto Biológico, Laboratório de Química Ambiental, São Paulo, SP (e-mail: rprisco@biologico.sp.gov.br).

*** Químico, Doutor em Química Ambiental, Instituto Biológico, Laboratório de Ecologia de Agroquímicos, São Paulo, SP (e-mail: luchini@biologico.sp.gov.br).

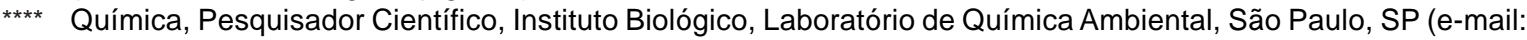
savoy@biologico.sp.gov.br).

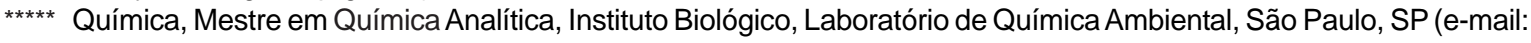
vieiraeliane@biologico.sp.gov.br).

*****Bióloga, Doutora em Ecologia, Instituto Biológico, Laboratório de Toxicologia, São Paulo, SP (e-mail: nakagawa@biologico.sp.gov.br). 


\section{INTRODUÇÃO}

As grandes cidades brasileiras, de modo geral, apresentam moradias precárias, em locais nem sempre adequados à construção civil. Também os sistemas de saneamento e de distribuição de água potável, de tratamento de esgotos e coleta do lixo, muitas vezes, mostram-se ineficientes para atender à demanda. Essas características geram condições propícias ao estabelecimento e desenvolvimento de animais sinantrópicos, muitos dos quais relacionados com diversas patogenias humanas (BRASIL, 2002; MORIN et al., 1989; VIEIRA, ALMEIDA e ALMEIDA, 1994). Os ratos merecem destaque entre as espécies encontradas nas cidades brasileiras, principalmente o Rattus norvegicus que está direta ou indiretamente relacionado com graves patogenias humanas, como a leptospirose (ALMEIDA et al., 1994; COMER, PADDOCK e CHILDS, 2001; RAO et al., 2003; SILVA et al., 2003). O controle desses roedores deve ocorrer por meio do manejo ambiental, embora muitas vezes seja necessário o uso de produtos hidroxicumarínicos (BRASIL, 2002). Entre as várias moléculas de hidroxicumarínicos utilizadas, o brodifacum tem se destacado e, consequentemente causado importantes problemas ambientais (BRITO et al., 2005; WALKER et al., 2008).

O brodifacum, pó branco com ponto de fusão entre 201 e $205^{\circ} \mathrm{C}$ (EPA, 2005), é pouco hidrossolúvel e estável à hidrólise. Quando em solução, é degradado pela luz ultravioleta (UV) (TOXNET, 2004). Seu coeficiente de partição octanol-água (log Kow=8,5) e seu fator de bioacumulação (FBC) estimado sugerem que o potencial de bioconcentração em organismos aquáticos seja alto. Já seu coeficiente de partição carbono orgânico (Koc $\left.=7,5 \times 10^{6}\right)$ estimado indica que o composto é imóvel em solo, com meia-vida de 14 dias em solo aeróbico (EPA, 2005). Apresenta-se comercialmente formulado como péletes e bloco parafinado. Por resistir melhor à umidade, o bloco parafinado é colocado preferencialmente em bueiros (BRASIL, 2002), preso por arame de modo a evitar contato permanentemente com a água, permitindo, ao mesmo tempo, que o animal tenha acesso à isca e a ingira. O bloco permanece nesse meio até que o animal o coma, o que nem sempre ocorre. Segundo informação de Agentes de Zoonoses que manipulam o produto, algumas vezes, os blocos ainda se encontram no local após 10 dias com aparência, coloração e textura alteradas surgindo a dúvida da real concentração do princípio ativo na formulação (SOUZA e COSTA, 2008).

Vários fatores influenciam o comportamento dos raticidas no ambiente como, por exemplo, as suas propriedades físicas e químicas, o tipo de formulação, a quantidade, frequência e modo de sua aplicação, as características abióticas e bióticas do meio e as condições meteorológicas do local de aplicação. De modo geral, quanto mais longa a permanência do composto no ambiente mais acentuada será a contaminação ambiental (AMORIN et al., 2002; RACKE, 1990). Além disso, pode ocorrer a degradação do composto em produtos até mais tóxicos e, dessa maneira provocar intoxicação e contaminação de organismos não alvos (PAPINI et al., 2005).

Os raticidas interferem no processo de coagulação sanguínea e exercem ação vasodestrutiva, levando ao extravasamento sanguíneo (LARINI, 1999). Embora a ação dos raticidas hidroxicumarínicos no organismo seja bastante conhecida, existem poucas informações sobre o comportamento ambiental do i.a. brodifacum formulado como bloco parafinado. Assim, procurou-se determinar a estabilidade da molécula no produto formulado como bloco parafinado, quando exposta às condições ambientais no meio urbano. 


\section{MATERIAL E MÉTODOS}

O brodifacum grau técnico, com 99,5\% $\mathrm{m} / \mathrm{m}$ de pureza química, foi adquirido da empresa Chem Service e utilizado no preparo de soluções padrão para estabelecimento das condições de análise. O produto comercial bloco parafinado foi cedido pela Unidade de Vigilância Ambiental da Vila Mariana e também adquirido em lojas especializadas para uso nos ensaios experimentais e realização dos testes de recuperação do i.a., bem como colocados na área externa do Instituto Biológico e nos bueiros da região de Vila Mariana.

\subsection{ESTABELECIMENTO DAS CONDIÇÕES DEANÁLISE}

A detecção e a quantificação do i.a. grau técnico brodifacum foram realizadas por Cromatografia a líquido de alta eficiência (CLAE) em equipamento DIONEX UVD 170U/340U, equipado com detector ultravioleta-visível em comprimento de onda de $260 \mathrm{~nm}$, coluna fase não polar octadecil-sílica RP-18 de comprimento $250 \times 46 \mathrm{~mm}$, temperatura de trabalho de $22^{\circ} \mathrm{C}$ e fase móvel metanol:água:ácido acético glacial (88,5:10:1,5 v/v) com vazão de $1 \mathrm{~mL}$ min $^{-1}$ (PEREZ, 1982). Foram preparadas soluções de 1,$25 ; 2,5 ; 5,0$ e $10 \mu$ g i.a. $\mathrm{mL}^{-1}$ de acetonitrila no momento da utilização.

Para confirmação do pico também foram avaliadas a detecção e a quantificação em equipamento SHIMADZU LC-10AD, detector ultravioleta-visível em comprimento de onda de $260 \mathrm{~nm}$ e detector de fluorescência ex $318 \mathrm{~nm}$ e em $390 \mathrm{~nm}$, com coluna RP-18 $5 \mu \mathrm{m} \times 25 \mathrm{~cm}$ x 0,46 mm, fase móvel metanol:água:ácido acético glacial (88,5:10:1,5 v/v) com vazão de $0,7 \mathrm{~mL} \mathrm{~min}{ }^{-1}$. Soluções de 0,$4 ; 0,8 ; 1,6 ; 4,0 ; 8,0 ; 16 ; 20$ e $32 \mu \mathrm{g}$ i.a. $\mathrm{mL}^{-1}$ de acetonitrila foram preparadas e injetadas no cromatógrafo, sendo o ingrediente ativo identificado por UV-visível e por fluorescência.

A partir dos resultados obtidos, com as diferentes concentrações de i.a., efetuou-se a curva padrão do ativo brodifacum com limites de detecção e de quantificação estabelecidos pelo método de MEIER e ZÜND (1993)

\subsection{AVALIAÇÃO DA PRESENÇADE BRODIFACUM FORMULADO COMO BLOCO PARAFINADO EM ANIMAIS DE EXPERIMENTAÇÃO}

Antes do início do ensaio, o projeto foi submetido ao Comitê de Ética e Pesquisa com Animais do Instituto Biológico - CETEA IB (nº 049/08). Foram utilizados ratos machos adultos e saudáveis da linhagem Wistar, fornecidos pelo biotério da Faculdade de Medicina da Universidade Federal de São Paulo (UNIFESP), com peso aproximado de $300 \mathrm{~g}$. Os animais foram colocados em gaiolas separadas, 1 animal por gaiola, e durante 7 dias ficaram no Laboratório de Toxicologia, antes do início do experimento para adaptação às condições do local.

Seguindo metodologia adaptada da ANVISA (2004), 1 animal-controle e 5 animaisteste (identificados de 1 a 5) foram mantidos individualmente em gaiolas, com fornecimento de água à vontade, iluminação natural e em condições adequadas de higiene. O número de animais foi determinado pelo programa estatístico PRIMER (GLANTZ, 1992).

No primeiro dia do estudo foram fornecidas ração e água aos animais e no segundo dia, a quantidade de ração consumida foi registrada e o bloco parafinado com brodifacum fornecido. No terceiro dia, a quantidade de bloco parafinado consumida foi registrada e o bloco substituído por ração. Nos dias subsequentes até o $14^{\circ}$ dia, a quantidade de ração consumida foi registrada diariamente e o comportamento e a aparência dos animais observados. 
A data da morte de cada animal foi registrada e o cadáver avaliado quanto à presença de pontos hemorrágicos nos tecidos.

\subsection{ESTUDO DE DISSIPAÇÃO - BRODIFACUM}

Os ensaios para avaliação da dissipação do i.a brodifacum na formulação bloco parafinado foram realizados em área externa do Instituto Biológico de São Paulo, cercada e identificada quanto ao risco químico. Também foram colocados 100 (cem) blocos parafinados em bueiros na região da Vila Mariana.

Foram construídos sistemas constituídos por tubo de policloreto de vinila (PVC) com $20 \mathrm{~cm}$ de diâmetro, cortado em pedaços com $25 \mathrm{~cm}$ de altura. No fundo de todos os tubos colocou-se uma tampa de PVC acrescida de borracha isolante. Cada tubo foi furado a aproximadamente $5 \mathrm{~cm}$ do fundo, no qual colocou-se um pedaço de mangueira de poliuretano de 2,0 $\mathrm{mm} \times 0,5$ polegadas e $50 \mathrm{~cm}$ de comprimento. Os sistemas foram numerados de 1 a 30, dispostos aleatoriamente sobre os estrados e cobertos com tela de arame. Foram acopladas as mangueiras de poliuretano com $50 \mathrm{~cm}$ de comprimento que saíam de cada sistema em garrafões numerados de 1 a 30 para coleta de água correspondente. Fixou-se um bloco parafinado em cada sistema por meio de arame galvanizado, preso à tela de cobertura (Figura 1). Semanalmente, durante 10 semanas, 3 sistemas foram sorteados e desmontados, retirandose os blocos que foram identificados e armazenados em congelador $\left(-10^{\circ} \mathrm{C}\right)$ até extração e análise. Os sistemas foram mantidos nos meses de dezembro, janeiro e fevereiro, correspondendo ao período de maior utilização de blocos parafinados nos bueiros. No mesmo período, 100 blocos contendo o i.a. foram colocados em bueiros da região da Vila Mariana e 3 amostras aleatórias retiradas semanalmente para análise, sendo também verificada a manutenção dos blocos colocados inicialmente em cada bueiro.

Para extração do i.a., as amostras foram maceradas e uma alíquota de $8 \mathrm{~g}$ extraída com $25 \mathrm{~mL}$ de metanol+diclorometano+ácido acético $(9+0,75+0,25)$ em ultrassom por 1 hora a $40^{\circ} \mathrm{C}$. Os extratos foram filtrados em papel filtro $(2,0 \mu \mathrm{m})$ e analisados por cromatografia a líquido de alta eficiência. A presença de i.a. em cada amostra foi apresentada como porcentagem do detectado no $\mathrm{T}_{\text {zero }}$.

\section{FIGURA 1 - SISTEMAS (A) EM QUE FOI COLOCADA A ISCA RATICIDA FORMULADA COMO BLOCO PARAFINADO FIXADA POR MEIO DE ARAME À TELA DE COBERTURA (B)}

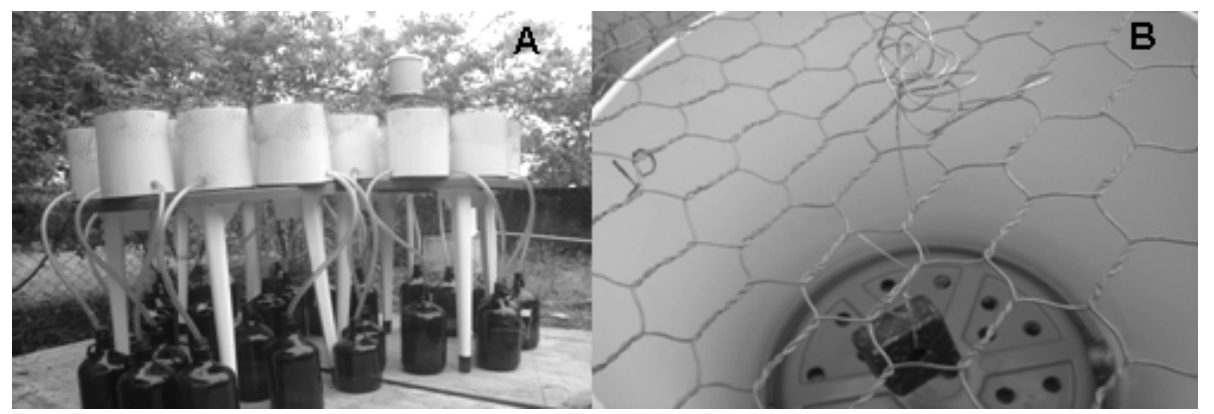

As condições ambientais, pluviosidade e temperatura, foram monitoradas por meio de pluviômetro e termômetro de máxima e mínima, dispostos no local do experimento. A medição da pluviosidade ocorreu uma vez por semana, sendo as temperaturas mínimas e máximas verificadas diariamente no local do experimento, exceto nos fins de semana e feriados. 


\section{RESULTADOS}

\subsection{ESTABELECIMENTO DAS CONDIÇÕES DE ANÁLISE}

Os limites de quantificação $\left(0,6136 \mu\right.$ i.a. $\left.\mathrm{mL}^{-1}\right)$ e de detecção $\left(0,3137 \mu\right.$ i i.a. $\left.\mathrm{mL}^{-1}\right)$ do método foram estabelecidos estatisticamente por análise de regressão com limite de confiança 95\% (MEIER e ZÜND, 1993). A curva de calibração em equipamento DIONEX, obtida a partir das soluções-padrão apresentou linearidade na faixa de concentração de 1,25 a 10,0 $\mu \mathrm{g} \mathrm{mL}^{-1}$, coeficiente de correlação $(\mathrm{r})$ de 0,9995 e coeficiente de determinação $\left(r^{2}\right)$ de 0,9992. O tempo de retenção do i.a foi de 10,2 minutos nas condições apresentadas (Figura 2).

A curva de calibração estabelecida em equipamento SHIMADZU LC-10AD apresentouse linear nas concentrações de 0,8, a $32 \mu \mathrm{g}$ i.a. $\mathrm{mL}^{-1}$.

A Figura 3 apresenta o cromatograma com as curvas de brodifacum em detector de fluorescência e de UV-visível.

FIGURA 2 - CROMATOGRAMAS DO BRODIFACUM COM 1,25; 2,5; 5,0 E 10,0 $\mu \mathrm{g}$ I.A. $\mathrm{mL}^{-1}$ OBTIDOS EM EQUIPAMENTO DIONEX, EQUIPADO COM DETECTOR ULTRAVIOLETA-VISÍVEL DIONEX UVD 170U/340U

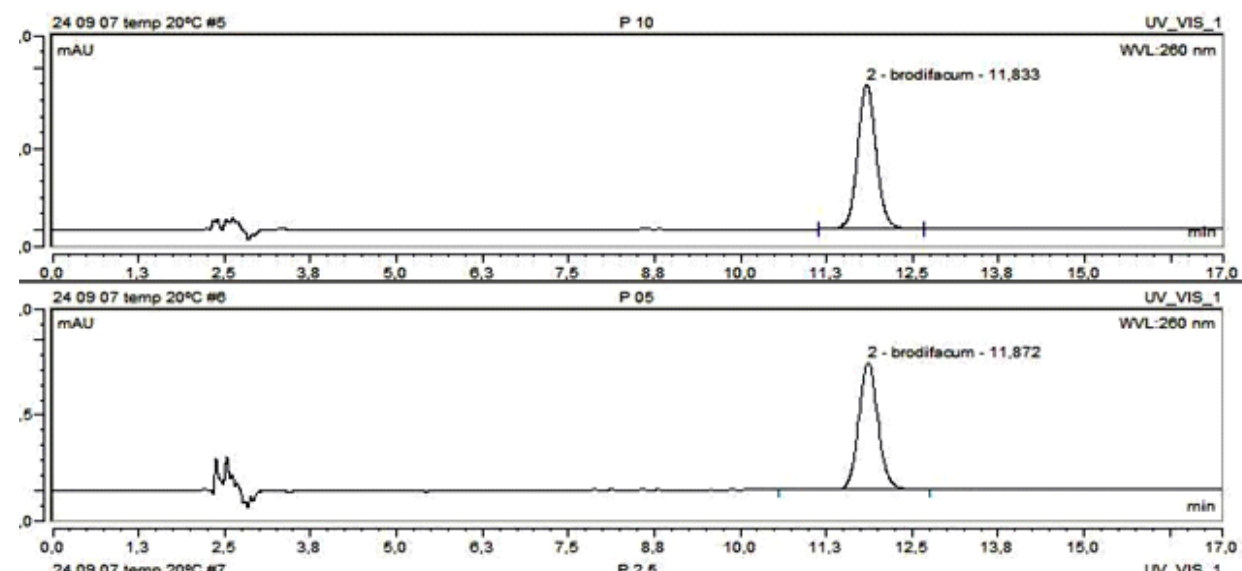

$10 \mu \mathrm{g}$ i.a.imL

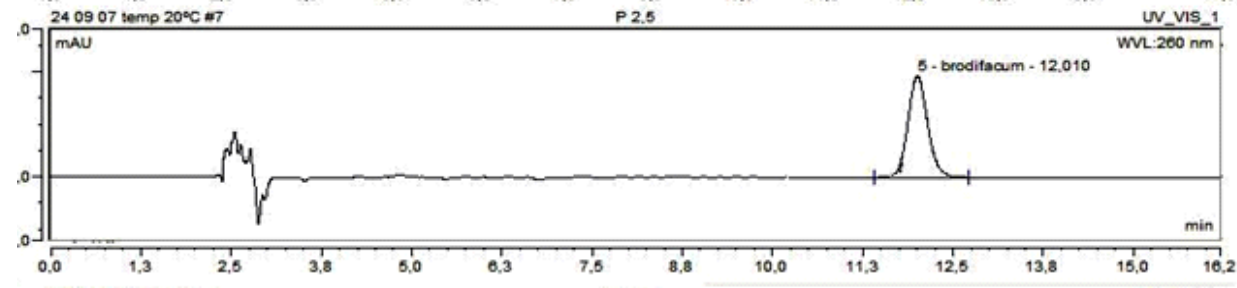

$2,5 \mu \mathrm{g}$ i.a./mL

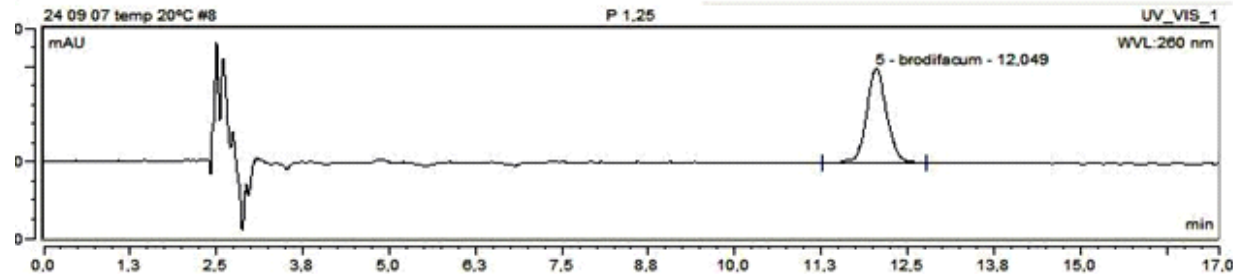

$5 \mu \mathrm{g}$ i.a.jmL

$1,25 \mu \mathrm{g}$ i.a./mL

Os valores ao lado dos gráficos correspondem à concentração do i.a. presente na amostra. Os cromatogramas mostram que até $1,25 \mu \mathrm{g}$ do ingrediente ativo $\mathrm{mL}^{-1}$ de solvente foi possível detectar e quantificar a concentração presente na solução. 


\section{FIGURA 3 - CROMATOGRAMA OBTIDO A PARTIR DE SOLUÇÃO COM O I.A BRODIFACUM EM CINZA SOB DETECTOR DE FLUORESCÊNCIA E EM PRETO SOB UV-VISÍVEL}

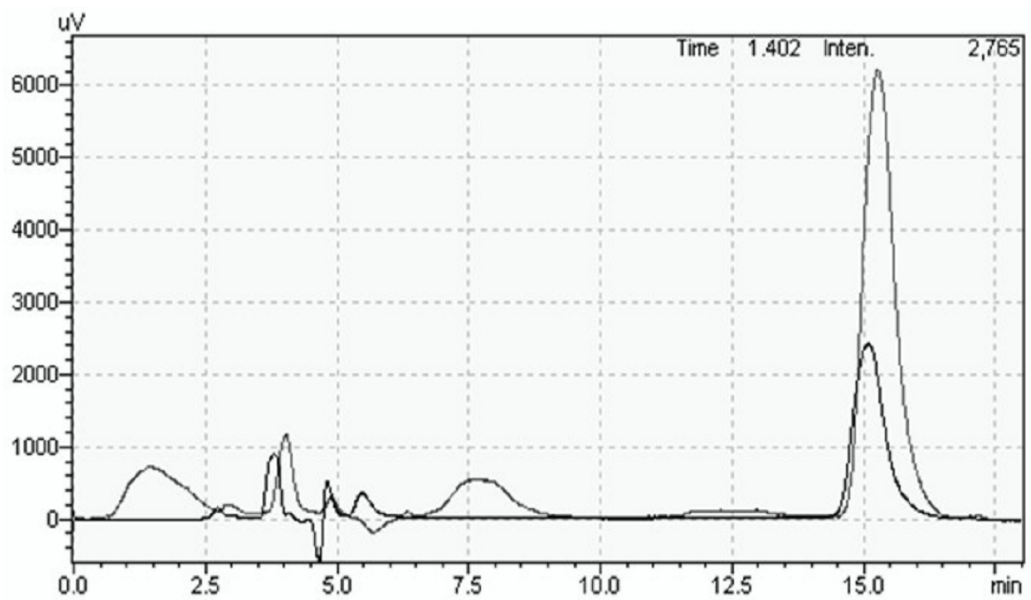

\subsection{AVALIAÇÃO DA PRESENÇA DE BRODIFACUM FORMULADO COMO BLOCO PARAFINADO EM ANIMAIS DE EXPERIMENTAÇÃO}

O ensaio revelou a presença do i.a. nos blocos parafinados do lote utilizado no estudo. $O$ consumo de ração entre os animais-teste variou entre 5,3\% e 6,5\% do peso, mostrando que os ratos estavam saudáveis e apresentavam condições físicas similares. Já o consumo de ingrediente ativo, presente na isca raticida e medido a partir da quantidade de bloco parafinado ingerido pelo animal, mostrou ampla variação. $O$ animal-teste 3 consumiu apenas $0,6 \%$ de seu peso enquanto que o animalteste 1 consumiu 4,8\%.

Constatou-se a presença do i.a. nos blocos parafinados utilizados no experimento, pois os animais morreram alguns dias após sua ingestão. Também foi constatada forte correlação entre a quantidade de i.a. consumida pelo animal e o número de dias após a ingestão em que ocorreu a morte (Figura 4). Quanto maior a quantidade do produto bloco parafinado consumido, e consequentemente maior a quantidade de i.a., mais rápido ocorreu a morte do animal.

\section{FIGURA 4 - CORRELAÇÃO ENTRE A PORCENTAGEM DE I.A. PRESENTE NO PRODUTO COMERCIAL INGERIDO PELO ANIMAL E O NÚMERO DE DIAS APÓS A INGESTÃO EM QUE OCORREU A MORTE}

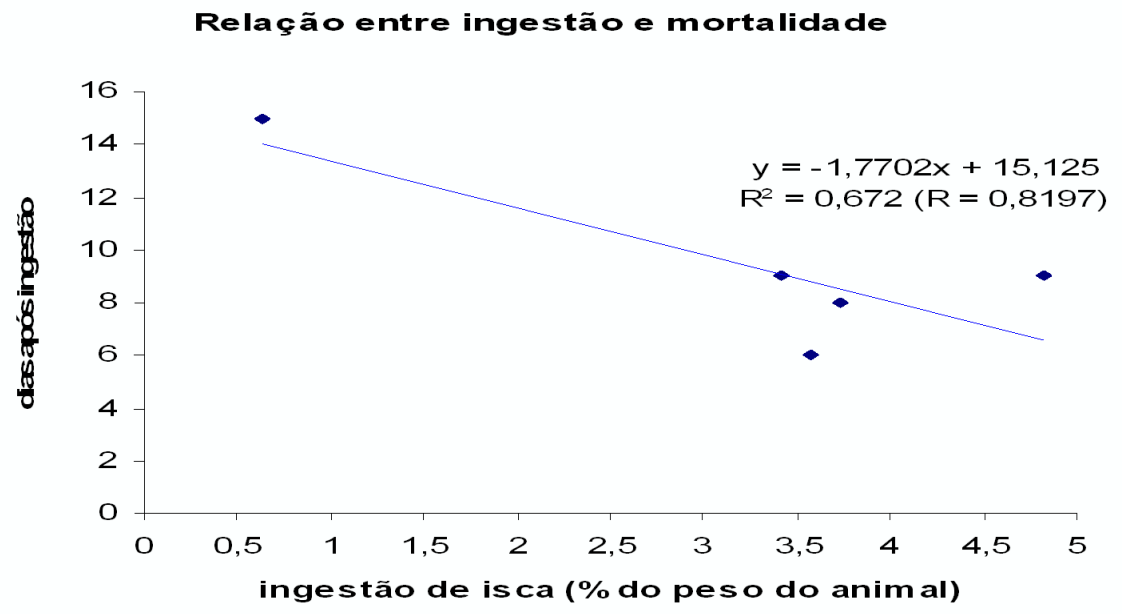




\subsection{ESTUDO DE DISSIPAÇÃO - BRODIFACUM}

Alguns blocos parafinados soltaram-se do arame de fixação após a terceira semana de exposição às condições ambientais. A variação de temperatura durante o período de exposição é apresentada na Figura 5, mostrando pequena variação na temperatura mínima e maior variação na temperatura máxima entre a $2^{\mathrm{a}}$ e a $4^{\mathrm{a}}$ semanas. A análise dos extratos das amostras $T_{\text {zero }}$ revelou que houve $100 \%$ de recuperação do i.a. e as porcentagens de recuperação durante as 10 semanas dos blocos mantidos nos sistemas do Instituto Biológico são apresentadas na Tabela 1, mostrando que houve cerca de $50 \%$ de dissipação do i.a. após 10 semanas de exposição. Dos 100 blocos colocados nos bueiros, somente 58\% foram recuperados íntegros ou parcialmente roídos e sua análise química revelou a presença de $100 \%$ do i.a.

\section{DISCUSSÃo}

O ensaio com animais demonstrou a presença do i.a. brodifacum nos blocos parafinados, uma vez que os animais morreram alguns dias após ingestão do produto. A necropsia dos animais submetidos ao experimento revelou a presença de hematomas e de sufusões hemorrágicas nos órgãos internos. Assim, o ensaio indicou a presença do i.a. de forma indireta por meio da morte por hemorragia dos animais testados.

\section{FIGURA 5 - VARIAÇÃO DE TEMPERATURA MÁXIMA (EM CINZA) E MÍNIMA (EM PRETO) AO LONGO DAS 10 SEMANAS DE EXPOSIÇÃO DOS BLOCOS PARAFINADOS NOS SISTEMAS MONTADOS EM ÁREA EXTERNA DO INSTITUTO BIOLÓGICO SOB CONDIÇÕES AMBIENTAIS}

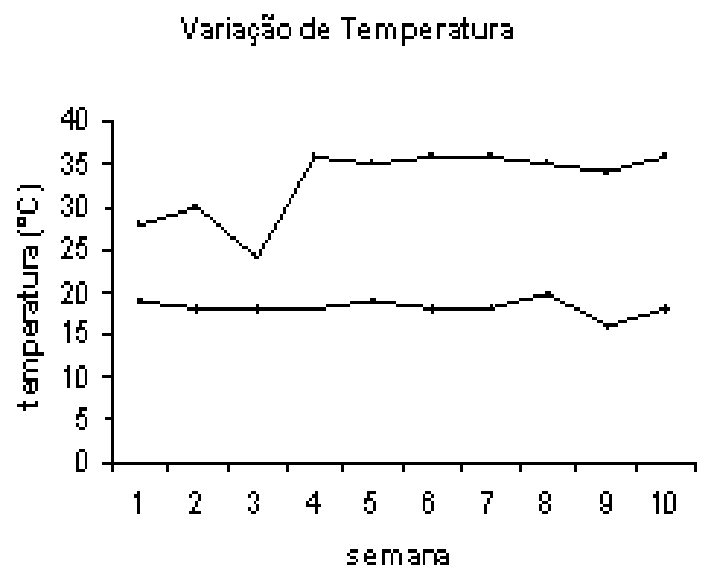

Dos 100 blocos colocados em bueiros da região da Vila Mariana no ensaio de dissipação do brodifacum, cerca de 50\% foram recuperados para análise laboratorial. O restante não se encontrava fixado ao arame, havendo dúvida se foram consumidos pelos animais ou se foram soltos do arame de fixação. Nos sistemas montados no Instituto Biológico, isolados de modo a impedir o acesso de animais, muitos blocos soltaram-se do arame em cerca de 2 a 3 semanas após sua fixação, demonstrando que o produto comercial apresentava baixa resistência física. Possivelmente, a elevada temperatura (máxima de até $30^{\circ} \mathrm{C}$, Figura 5) contribuiu para o derretimento da parafina, levando à soltura do arame de fixação. Essa característica dificulta o trabalho em campo, pois ao verifica devido ao 
derretimento da parafina. Além disso, os blocos ao se soltarem do arame podem entrar em contato direto com a água prejudicando o acesso dos roedores ao produto e causar contaminação do meio. Segundo informações de Agentes de Zoonoses que trabalham com raticidas no verão, muitas vezes, os blocos parafinados contendo bromadiolona ou brodifacum ficam moles dentro do veículo de transporte e não podem ser utilizados (SOUZA e COSTA, 2008). Segundo a RDC 326, de 09/11/2005, por ocasião da solicitação para registro de produtos desinfestantes domissanitários, entre os quais se encontram os raticidas, deverão ser apresentados os dados químicos e toxicológicos, resultados dos testes de eficácia sobre as pragas indicadas no painel principal do rótulo e metodologia de análise do(s) princípio(s) ativo(s) e sua determinação no produto formulado (ANVISA, 2005). A baixa resistência dos blocos parafinados usados neste estudo mostra que a temperatura pode interferir diretamente na eficácia do produto, pois uma vez derretido o bloco e em contato com a água deixa de ser atraente para o animal. Também aumenta o risco de contaminação ambiental, uma vez que é carreado para o sistema de águas pluviais. Assim, destaca-se a importância de testes físicos normalizados à semelhança do que existe com os produtos agrotóxicos, objetivando o controle de qualidade e a verificação da eficácia do produto aliada às suas características físico-químicas.

Os resultados das análises químicas cromatográficas revelaram que nos sistemas montados no Instituto Biológico, a presença do i.a. diminuiu gradativamente ao longo das 10 semanas de estudo chegando a 50\% da concentração inicial. Nos blocos provenientes dos bueiros, a concentração do i.a. manteve-se em 100\% durante todo o período estudado (Tabela 1). Talvez os resíduos orgânicos e inorgânicos, carreados pela água sobre os blocos nos bueiros, possam ter protegido o ingrediente ativo da ação de fatores atuantes na sua degradação, como luz e água. A baixa taxa de fixação dos blocos aos arames e a permanência do brodifacum por 10 semanas são fatores importantes a considerar na utilização desse produto, pois o ingrediente ativo pode ser carreado para a rede de coleta pluvial e contaminar os ambientes aquáticos expostos.

\section{TABELA 1 - RECUPERAÇÃO DO I.A. BRODIFACUM DO BLOCO PARAFINADO EXPOSTO POR 10 SEMANAS ÀS CONDIÇÕES AMBIENTAIS - SISTEMAS INSTITUTO BIOLÓGICO}

SEMANA

Tzero

T1

T2

T3

T4

T5

T6

T7

T 8

T9

T10
\% RECUPER AÇÃO 


\section{CONCLUSÃO}

O produto bloco parafinado contendo o ingrediente ativo brodifacum do lote utilizado no estudo mostrou baixa resitência à temperatura, embora tenha sido eficaz para a mortalidade dos ratos expostos a condições laboratoriais.

A concentração do ingrediente ativo brodifacum formulado como bloco parafinado diminuiu em relação à concentração inicial nos sistemas controlados, mas não se alterou nos blocos mantidos em bueiros durante as 10 (dez) semanas de estudo.

\section{ABSTRACT \\ EVALUATION OF THE DISSIPATION IN THE ENVIRONMENT OF RODENTICIDE BRODIFACOUM FORMULATED AS PARAFFINED BLOCK}

In the chemical control of rodents in the São Paulo city - Brazil, mainly rats, paraffined blocks containing the active ingredient (a.i.) brodifacoum, are put in gutters being subject to the action of the bad weather that could cart alterations in a.i. and transfer to other environments. Aiming to verify those possibilities, experimental systems with paraffined blocks were mounted simulating the field conditions. Also 100 blocks were put at gutters monitored weekly. Three blocks were weekly collected, for 10 weeks, of the systems and of the gutters, and analyzed by high performance liquid chromatography. The obtained data were used for the evaluation of the dissipation of active ingredient. The results pointed the stability of the molecule under real application environmental conditions, because $100 \%$ of a.i. was recuperated after exposition of the block. Although, in the experimental systems, about $50 \%$ of the blocks unfastened from the fixation wire to the fifth week of exhibition and the recovery of a.i. decreased gradually along the 10 weeks of the study, reaching $50 \%$ of the initial concentration.

KEY-WORDS: RODENTICIDE - PERSISTENCE; HYDROXYCUMARIN; ENVIRONMENTAL CONTAMINATION.

\section{REFERÊNCIAS}

1 ALMEIDA, L.P.; MARTINS; L.F.S.; BROD, C.S.; GERMANO, P.M.L. Levantamento soroepidemiológico de leptospirose em trabalhadores do serviço de saneamento ambiental em localidade urbana da região sul do Brasil. Revista de Saúde Pública, v. 28, p.1-9, 1994.

2 AMORIM, M.J.B.; SOUSA, J.P.; NOGUEIRA, J.A.; SOARES, A.M.V.M. Bioaccumulation and elimination of ${ }^{14} \mathrm{C}$-lindane by Enchytraeus albidus in artificial (OECD) and a natural soil. Chemosphere, v. 49, p.323329, 2002.

3 ANVISA. Agência Nacional de Vigilância Sanitária. Manual de protocolos para testes de eficácia de produtos desinfestantes. 2004. 45 p. Disponível em: www.anvisa.gov.br/reblas/link_desinfestantes.pdf. Acesso em: 25/mar./2008.

4 ANVISA. Agência Nacional de Vigilância Sanitária. Resolução da Diretoria Colegiada n 326, de 09 de novembro de 2005. Aprova o Regulamento técnico para produtos desinfestantes domissanitários. Disponível em: http://www.anvisa.gov.br/legis/index.htm. Acesso em:14/mar./2008

5 BRASIL. Ministério da Saúde. Manual de controle de roedores. Brasília: Fundação Nacional da Saúde, 2002. $132 \mathrm{p}$.

6 BRITO, M.F.; SEIXAS, J.N.; JABOUR, F.F.; ANDRADE, G.B.; CUNHA, B.R.M.; FRANÇA, T.N.; PEIXOTO, P.V. Sobre um surto de envenenamento por derivado cumarínico em bovinos. Pes. Vet. Bras., v. 25, n. 3, p. 143-149, 2005.

7 COMER, J.A.; PADDOCK, C.D.; CHILDS, J.E. Urban zoonosis caused by Bartonella, Coxiella, Ehrlichia and Rickettsia species. Vector Borne Zoonotic Diseases, v. 1, p. 91-118, 2001.

8 EPA. Environmental Protection Agency. Reregistration eligibility decision (RED). July, 1998. Disponível em: http://www.epa.gov/REDs/2100red.pdf Acesso em: 25/mar./2005. 
9 GLANTZ, S.A. Primer of biostatistics. San Francisco: McGraw-Hill, 1992.

10 LARINI L. Toxicologia dos praguicidas. São Paulo: Manole,1999.

11 MEIER, P.C.; ZÜND R.E. Statistical methods in analytical chemistry. New York: John Wiley \& Sons, 1993.

12 MORIN, M.F.; MERLET, N.; DORE M.; LECHEVIN J.C. Analyse de résidus de bromadiolone (rodenticide anticoagulant) dans l'eau et les tissus animaux. Analusis, v. 7, p. 526-531, 1989.

13 PAPINI, S.; HOMEM DE MELO, M.H.S.; OLIVEIRA, D.C.; ANDRÉA, M.M.; DAL BOM, M.G.; CREOLEZ, E.F.A; LUCHINI, L.C. O uso de inseticidas e raticidas no controle da fauna sinantrópica no município de São Paulo: contaminação da população e do ambiente? Revista de Vigilância Sanitária, v. 1, p. 174-179, 2005.

14 PEREZ, R.L. Simplified extraction procedure for the determination of brodifacoum in wheat baits by high-performance liquid chromatography. Analyst, v. 107, n. 1277, p. 968-971, 1982.

15 RACKE, K.D. Pesticides in the soil microbial ecosystem. In: RACKE, K.D.; COATS, J.R. (Org). Enhanced biodegradation of pesticides in the environment. Washington: American Chemical Society, 1990. p.1-12.

16 RAO, S.R.; GUPTA, N.; BHALLA, P.; AGARWAL, S.K. Leptospirosis in Indian and the rest of the world. Brazilian Journal of Infectious Diseases, v. 3, p. 178-193, 2003.

17 SILVA, H.R.; TAVARES-NETO, J.; BINA, J.C.; MEYER, R. Leptospiral infection and subclinical presentation among children in Salvador, Bahia. Rev. Soc. Bras. Med. Trop., v. 36, p. 227-233, 2003.

18 SOUZA, J.L.; COSTA, M.R. Atividades desenvolvidas por Agentes de Zoonoses no controle de roedores. Entrevista realizada em 14 de janeiro de 2008, na Supervisão de Vigilância em Saúde (SUVIS), Vila Mariana-Jabaquara em São Paulo/SP.

19 TOXNET. Toxicology Data Network. United States National Library of Medicine. Brodifacoum. Disponível em: http://toxnet.nlm.nih.gov/cgi-bin/sis/search. Acesso em: 29/mar/2004.

20 WALKER, L.A.; TURK, K.A.; LONG, S.M.; WIENBURG, C.L.; BEST, J.; SHORE, R.F. Second generation anticoagulant rodenticides in tawny owls (Strix aluco) from Great Britain. Sci. Total Environ., v. 392, n. 1, p.93-98, 2008.

\section{AGRADECIMENTOS}

Este trabalho é parte do projeto avaliação da dissipação no ambiente de raticidas hidroxicumarínicos nas formulações granulado, pó e parafinado usados para desratizações referênciais, desenvolvido com financiamento FAPESP (projeto no 05/53829-6) 